\title{
Effect of Magnetic Particles on Structural Changes and Magneto-Optical Behavior of Liquid Crystal
}

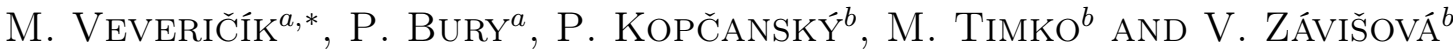 \\ ${ }^{a}$ Department of Physics, Žilina University, Univerzitná 1, 01026 Žilina, Slovakia \\ ${ }^{b}$ Institute of Experimental Physics, Slovak Academy of Sciences, Watsonova 47, 04001 Košice, Slovakia

\begin{abstract}
Structural changes in the nematic liquid crystal 6 CHBT doped with a low concentration $\left(1 \times 10^{-4}, 5 \times 10^{-4}\right)$ of magnetic nanorods induced by weak magnetic field in the increasing/decreasing mode, as well as in pulsed regime are investigated using surface acoustic waves and magneto-optical effect. The responses of both surface acoustic waves attenuation and optical transmission of the linearly polarized laser beam $(532 \mathrm{~nm})$ passing through the sample were investigated. The role of the concentration of magnetic nanorods on structural changes and corresponding magneto-optical behavior concerning the system stability and switching time under applied field was determined. The obtained results of both methods confirmed an effective orientational coupling between particles' magnetic moments and liquid crystal molecules and showed the effectivity of measurements relying on suspension stability and switching processes.
\end{abstract}

DOI: 10.12693/APhysPolA.137.967

PACS/topics: liquid crystal, nanorods, magneto-optics, SAW

\section{Introduction}

Nematic liquid crystals (NLCs) are well known as a thermodynamically stable state of matter represented by orientational molecular order with the long molecular axis as the preferred direction called the director $(\boldsymbol{n})$. The director $\boldsymbol{n}$ orientation is constant throughout the sample in the absence of an external field or certain boundary conditions. The external field (e.g., magnetic field) can change the orientation of the director after the threshold field is reached. By adding specifically chosen nanoparticles as dopant, the modification of some properties can be achieved. This modification can be observed as the change of the threshold field, the shift of the nematic-isotropic phase transition $[1,2]$.

Due to the anisotropy of diamagnetic susceptibility, the magnetic fields necessary to reorient or align 6CHBT liquid crystals molecules must reach high levels of magnetic flux density $(B>1 \mathrm{~T})$. The enhancement of the magnetic susceptibility of NLC was described as an idea of ferronematic liquid crystals that behave like magnetically active anisotropic fluids [3, 4]. A rigid anchoring is expected in the case when $\boldsymbol{m} \| \boldsymbol{n}$, where the director $\boldsymbol{n}$ denotes a preferential direction of the nematic molecules and the unit vector $\boldsymbol{m}$ represents the orientation of the magnetic moment of the magnetic particles, such compound would result into specific ferromagnetic behavior.

As we have recently shown, surface acoustic waves (SAW) spectroscopy is a useful nondestructive method for characterization of some NLC properties especially of structural changes in external fields [5]. The orientational transitions can be studied also by electro-optic and

*corresponding author; e-mail: vevericik@fyzika.uniza.sk magneto-optic techniques. It was found that variation in the field-assisted structural changes can be accompanied by oscillations of light intensity that could be attributed to the orientational redistribution of the nematic director $\boldsymbol{n}$ from uniform alignment [6,7]. The magneto-optical effect was investigated in lyotropic LCs doped with ferrofluid in their isotropic phase [8] and nematic LCs doped with ferromagnetic particles as well [9]. Some nematics demonstrate a memory effect - the residual transmittance after the field is switched off $[10,11]$. It should be noticed that the reorientation of NLC host was expected to be completely determined by the properties of ferromagnetic nanoparticles rather than by the intrinsic diamagnetic properties of the nematics. In the contribution we present the study of effect of nanorods presence on structural changes in 6CHBT under magnetic field.

\section{Experimental details}

The ferronematic samples that we studied, were based on 6CHBT (4-(trans-4'- $n$-hexylcyclohexyl)isothiocyanatobenzene) a thermotropic NLC, where 6CHBT is a low-melting enantiotropic liquid crystal characterized by high chemical stability [12]. The temperature of the nematic-to-isotropic transition for the studied nematic is $T_{N I}=42.8^{\circ} \mathrm{C}$. Needle shaped goethite nanorods with a mean diameter of $6 \mathrm{~nm}$ and lengths from 100 to $250 \mathrm{~nm}$ were synthesized through decomposition of urea and the resulting precipitation of $\mathrm{Fe}^{2+}$ in oleic acid micelles. The morphology and size distribution of prepared nanorods were measured by transmission electron microscopy (TEM Tesla BS 500). Nanorods were added in two volume concentration into 6CHBT matrix $\left(1 \times 10^{-4}, 5 \times 10^{-4}\right)$ [13].

The SAW of frequency $10 \mathrm{MHz}$ was generated by an interdigital transducer prepared on the $\mathrm{LiNbO}_{3}$ delay line, using the pulse modulator and receiver-MATEC 7700 . 
The second transducer was used for the reception of the surface wave passing along the interface of the delay line and sample. For the measurement of acoustic attenuation changes, MATEC attenuation recorder $2470 \mathrm{~A}$ or gated integrator boxcar averager SR 250 were used. The cell of NLCs compound $(D \approx 100 \mu \mathrm{m})$ was prepared at the center of the delay line between transducers and covered with glass plate. The sample holder was then placed between electromagnet poles. Stable temperatures could be achieved $\left( \pm 0.1^{\circ} \mathrm{C}\right)$ in the range from 5 to $50^{\circ} \mathrm{C}[4]$.

Magneto-optical investigations were done using linearly polarized laser beam (532 nm, $5 \mathrm{~mW})$ passing through the cell using optical triangular prism and polarizer. The whole LC cell holder was placed between poles of electromagnet. The cells were prepared from two glasses with $50 \mu \mathrm{m}$ gap and optically polished on both glass substrates to ensure initial parallel alignment of LC molecules. The intensity of transmitted light was recorded as a function of the magnetic field by a semiconductor photodetector connected to the computer via multimeter. The magnetic field can be controlled by a LabVIEW program and can work in increased/decreased mode $(80 \mathrm{mT} / \mathrm{min})$ or altered by a jump (pulsed) change [5].

\section{Results and discussion}

Structural changes in investigated NLC doped with magnetic nanorods $\left(\Phi=1 \times 10^{-4}, \Phi=5 \times 10^{-4}\right)$ induced by applied magnetic field were monitored using both measurements of SAW attenuation change $\Delta \alpha$ propagating along with the interface and optical transmission of light. As the initial intrinsic arrangement of NLC was supposed to have a predominately planar alignment, where the director was parallel to the surface of the delay line, the magnetic field was then applied perpendicular to them. The magneto-optical effect could be registered using parallel and crossed polarizers setup. Therefore, the light transmission was expressed as $I / I_{0}$ in the case of parallel polarizers, and as $\left(I-I_{0}\right) / I_{0}$ in the case of crossed polarizers. Here, $I$ is measured intensity and $I_{0}$ is the intensity of incident light passing through the doped NLC sample without applied magnetic field.

The comparison of structural changes under increasing magnetic flux density $(0-400 \mathrm{mT})$ is shown in Fig. 1 for both types of measurements and both concentrations. The changes in acoustic attenuation (Fig. 1a) show almost very weak attenuation change at lower fields followed by a faster increase and again the slowed-down increase that converges to the saturation. The change registered by acoustic attenuation practically starts at magnetic field of $100 \mathrm{mT}$ and for fields higher than $300 \mathrm{mT}$ the saturation occurs gradually.

The faster increase of the attenuation corresponds to the main process of reorientation of LC molecules that seems to be independent of the particle concentration.

The role of magnetic field strength on the optical transmission (Fig. 1b) for two different concentrations of
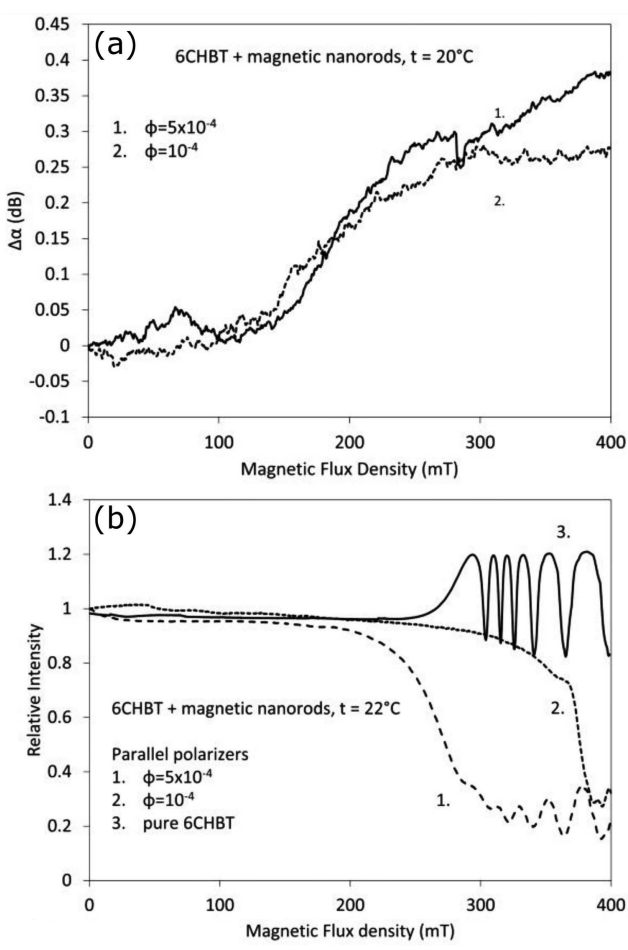

Fig. 1. Effect of applied magnetic flux density on (a) acoustic attenuation change, and (b) optical transmission of 6CHBT doped with magnetic nanorods $\left(\Phi=1 \times 10^{-4}\right.$ and $\left.\Phi=5 \times 10^{-4}\right)$ including pure 6 CHBT.

nanorods including pure 6CHBT shows that the higher concentration of nanorods leads to lower threshold field necessary to change the optical properties. The change of the magnetic threshold with increasing concentration is mainly influenced by the coupling between LC molecules and magnetic nanorod. This fact can partly coincide also with previous results due to the role of a process of particles aggregation [14].

Because of partial depolarization by the pure $6 \mathrm{CHBT}$ of the incident light [15], the observed response in crossed polarization state (Fig. 2), compared with previous one (Fig. 1b), indicates an increase of optical transmission after the same threshold field as in transmission decrease of the parallel polarizers arrangement. The change in correlative orientation between NLC molecules and light polarization plane gives rise to the existence of oscillations. Observed oscillations curves can be attributed to the fact that during the Fréedericksz transition the director of planar alignment sample undergoes a reorientation between two fixed directions, from planar to normal [16]. In the decreasing mode, a slight memory effect occurs with a minor shift of the threshold field value.

The dynamics of the switching processes for different values magnetic of flux density are shown in Fig. 3, where the effect of the applied field in pulsed regime (duration $120 \mathrm{~s}$ ) points out the structural changes dependent on the magnitude of the field (200, 300, and $400 \mathrm{mT}$ ). The duration time was chosen based on previous results 


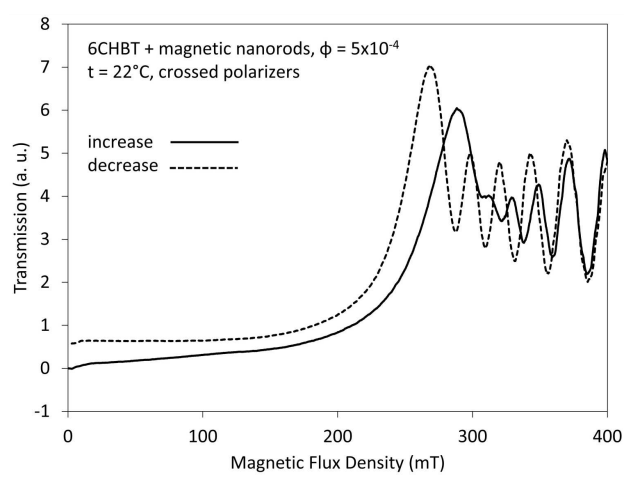

Fig. 2. Effect of applied increasing and decreasing magnetic flux on optical transmission properties with crossed polarizers of 6CHBT doped with magnetic nanorods $\left(\Phi=5 \times 10^{-4}\right)$.
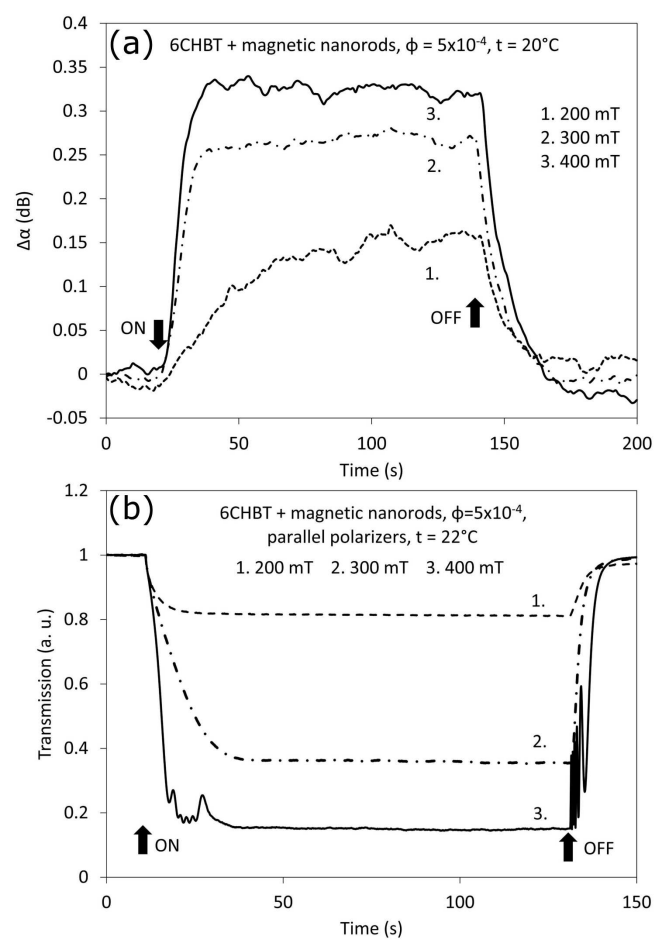

Fig. 3. Effect of applied pulsed magnetic flux density $(200,300,400 \mathrm{mT})$ on (a) acoustic attenuation change, and (b) optical transmission of 6CHBT doped with magnetic nanorods $\left(\Phi=5 \times 10^{-4}\right)$.

obtained from dependences of magnetic flux density (Fig. 1). Structural changes driven by the applied field could reach the saturated state during the selected time. No bias magnetic field was applied before starting the optical transmission measurement and vice versa for acoustic SAW measurements, so that suspensions appeared to be compensated ferronematic in that case. The magnetooptical time responses correspond to acoustic attenuation measurements for the same concentration. It is evident that the anchoring occurs due to the coupling of LC molecules and nanorods and ultimately ensures a stable magneto-optical response.

\section{Conclusions}

The study of structural changes and optical properties of NLC doped with nanorods is presented. By comparing the results of SAW spectroscopy and optical transmission we get reliable information about structural changes under the applied magnetic field in doped NLC and can determine the influence of doping process on properties of pure 6CHBT. Obtained results showed the change in the threshold field with increasing concentration for driving magnetic field and influence on the optical activity of NLC induced just by ferromagnetic dopants, resulting in the system stability in the pulsed regime of magnetic field. As was proposed, anchoring between NLC molecules and nanorods leads to orientational coupling, that can result in the compound stability and by that the stable response to the applied magnetic field.

\section{Acknowledgments}

This work was supported by VEGA projects $2 / 0016 / 17,1 / 0510 / 17$. Authors also would like to thank Mr. František Černobila for technical assistance.

\section{References}

[1] F. Li, O. Buchnev, C.I. Cheon, A. Glushchenko, V. Reshetnyak, Y. Reznikov, T.J. Sluckin, J.L. West, Phys. Rev. Lett. 97, 147801 (2006).

[2] R. Basu, A. Garvey, Appl. Phys. Lett. 105, 151905 (2014).

[3] F. Brochard, P.G. de Gennes, J. Phys. (France) 31 691 (1970).

[4] J. Liebert, A. Martinet, J. Phys. Lett. 40, 363 (1979).

[5] P. Bury, M. Veveričík, P. Kopčanský, M. Timko, Z. Mitróová, Physica E 103, 53 (2018).

[6] G. Si, Y. Zhao, E.S.P. Leong, Y.J. Liu, Materials 7, 1296 (2014).

[7] A.M. Prshin, V.A. Gunyakov, V.Y. Zyryanov, V.F. Shabanov, Int. J. Mol. Sci. 15, 17838 (2014).

[8] P.R.G. Fernandes, H. Mukai, I.M. Laczkowski, J. Magn. Magn. Mater. 289, 115 (2015).

[9] N. Podoliak, O. Buchnev, O. Buluy, G.D. Alessandro, M. Kaczmarek, Y. Reznikov, T.J. Sluckin, Soft Matter 7, 4742 (2011).

[10] A. Glushchenko, H. Kresse, V. Reshetnyak, Yu. Reznikov, O.V. Yaroshchuk, Liq. Cryst. 23, 241 (1997).

[11] G. Puchkovskaya, Yu. Reznikov, A. Yakubov, O.V. Yaroshchuk, A. Glushchenko, J. Mol. Struct. 404, 121 (1997).

[12] M. Szaleniec, R. Tokarz-Sobieraj, W. Witko, J. Mol. Model. 15, 935 (2009).

[13] P. Kopčanský, V. Gdovinová, S. Burylov, et al., J. Magn. Magn. Mater. 459, 26 (2018).

[14] P. van der Schoot, J. Chem. Phys. 117, 3537 (2002).

[15] A.W. Domanski, D. Budaszewski, M. Sierakowski, T.R. Wolinski, Opto-Electron. Rev. 14, 305 (2006). 